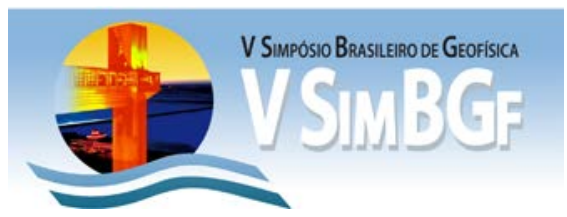

\title{
Estudo da sismicidade ao sul do Lineamento Pernambuco em 2010
}

H. C. de Lima Neto ${ }^{\star 1}$, J. M. Ferreira ${ }^{1 / 2}$, M.S. Assumpção ${ }^{2}$, A. F. do Nascimento ${ }^{12}$, F. H. R. Bezerra ${ }^{12}$, M. O. Sousa ${ }^{3}$, \&

E.A.S. Menezes².

1- Programa de Pós-Graduação em Geodinâmica e Geofísica, Universidade Federal do Rio Grande do Norte. Natal-RN.Brasil.

2- Departamento de Geofísica - UFRN.

2- Instituto de Astronomia, Geofísica e Ciências Atmosféricas - USP.

3- Departamento de Geologia - UFRN.

Copyright 2012, SBGf - Sociedade Brasileira de Geofísica

Este texto foi preparado para a apresentação no V Simpósio Brasileiro de Geofísica, Salvador, 27 a 29 de novembro de 2012. Seu conteúdo foi revisado pelo Comitê Técnico do V SimBGf, mas não necessariamente representa a opinião da SBGf ou de seus associados. É proibida a reprodução total ou parcial deste material para propósitos comerciais sem prévia autorização da SBGf.

\section{Resumo}

A região Nordeste do Brasil é uma das de maior atividade sísmica intraplaca no país (Berrocal et al. 1984; Assumpção, 1992,1993). Nos últimos quarenta anos, a atividade sísmica na região tem sido caracterizada por enxames de sismos que podem durar mais de 10 anos e por eventos que atingem magnitudes de até $5,2 \mathrm{~m}_{\mathrm{b}}$, na escala Richter.

As atividades sísmicas registradas por redes instaladas pelo Laboratório Sismológico da UFRN mostram o grande potencial sismogênico da região Nordeste do Brasil. Entre as principais áreas sismicamente ativas destaca-se o Agreste Pernambucano.

Nesta região, diversos eventos sísmicos vêm sendo relatados desde o século XIX e, nos últimos anos, tem se manifestado de forma mais intensa e numerosa (Ferreira et al., 2008; Lima Neto et al., 2009; Lopes et al., 2010). Além disso, esta região possui importantes estruturas tectônicas mapeadas, destacando-se o Lineamento Pernambuco, uma zona de cisalhamento de escala continental com $700 \mathrm{~km}$ de comprimento que aparece claramente na superfície (Davison, et al., 1995).

O presente estudo é o primeiro a ser realizado com uma rede local de estações sismográficas em áreas que estão ao sul do Lineamento Pernambuco. A principal feição geológica mapeada na região é a zona de cisalhamento Gravatá-Açu que possui tendência para NE e está localizada abaixo do Lineamento Pernambuco. Este estudo mostra os resultados obtidos da campanha realizada no período de 21/04/2010 a 22/09/2010.

\section{Introdução}

A atividade sísmica na região de Belém de Maria-PE teve início no dia 18/04/2010 quando ocorreu um sismo de magnitude $2.8 \mathrm{~m}_{\mathrm{R}}$ que provocou pânico no município de Belém de Maria-PE e no distrito Lajes de São José, pertencente ao município de Cupira-PE. Este evento chegou a ser registrado pela estação RCBR (Riachuelo$\mathrm{RN}$ ), distante 304 quilômetros da área epicentral (figura 1). As réplicas foram registradas pela estação GRBR (Gravatá - PE) e analisadas em tempo real; as análises preliminares através da estação GRBR mostraram que a atividade sísmica não estava concentrada em uma única área. Foi possível identificar a principal área localizada no município de cupira-PE e outra numa área ao sul do município de Lagoa dos Gatos.

No dia 21/04/2010 teve início a instalação de uma rede local estações de período curto na região de Belém de Maria que chegou a operar com 10 estações.

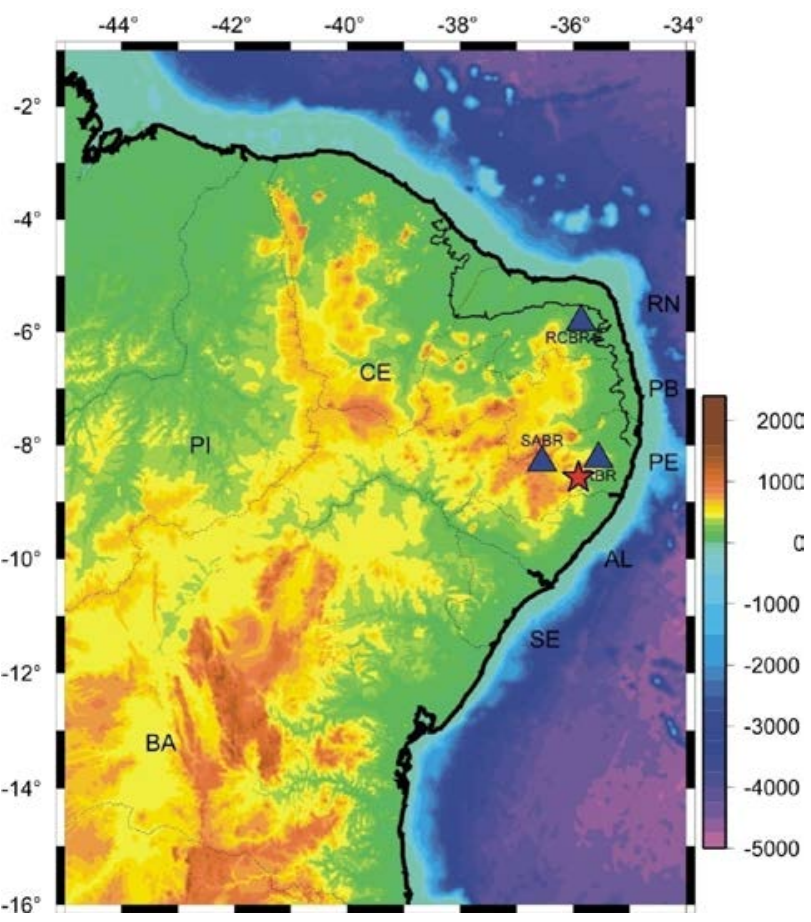

Figura 1 - A estrela vermelha indica a localização epicentral do evento de magnitude $2.8 m_{R}$ ocorrido em Cupira-PE no dia 18/04/2010. Os triângulos azuis indicam as estações RCBR (USGS-UFRN), SABR e GRBR ( as duas últimas pertencentes ao INCT-ET).

\section{Metodologia/ Problema Investigado}

Devido à participação do Laboratório Sismológico da UFRN no INCT de Estudos Tectônicos foi possível, neste estudo, a utilização das estações sismográficas de período curto do PEGBR (Pool de Equipamentos Geofísicos do Brasil). Foi utilizada uma rede sismográfica local composta por até dez estações providas de sismômetros de período curto modelo L4C3 (vertical, NS e EW, em cada estação) Todas as estações utilizaram o registrador Reftek130 (figura 2).

A rede local estações de período curto na região de Belém de Maria inicialmente contou com 6 estações e após duas semanas de operação foram instaladas outras 
4 estações (também de período curto) na região com o objetivo de tentar cercar a área ao Sul de Lagoa dos Gatos-PE, mas devido as intensas chuvas que antecederam a enchente que devastou os municípios da região não foi possível obter um arranjo de estações que cercasse os epicentros ao sul de Lagoa dos Gatos que foram registrados pela rede.

A atividade sísmica estudada neste trabalho esteve concentrada em três clusters, o principal no Distrito de Serra Verde (grupo 1) pertencente ao município de Cupira-PE, próximo a estação BMLJ, o segundo (grupo 2) fica mais ao norte de Cupira-PE, no município de Agrestina - PE e o terceiro (grupo 3), ao sul, no município de Lagoa dos Gatos - PE. Foram analisados os dados registrados pela rede de estações sismográficas (Rede BM) no período de Abril a Setembro de 2010.

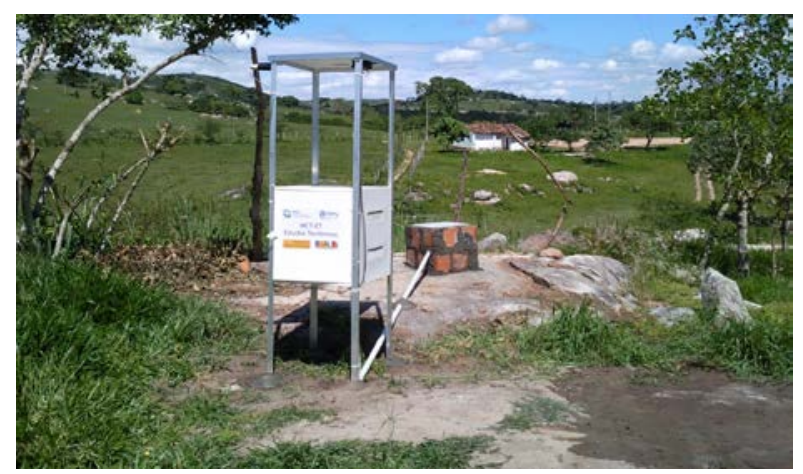

Figura 2- Rede BM2010, estação (BMCR) que esteve em operação no distrito de Cachoeira do Riachão em São Joaquim do Monte - PE. O sismômetro encontra-se dentro da estrutura de alvenaria. O registrador encontrase dentro da caixa branca.

O programa SAC (Tapley \& Tull, 1991) foi utilizado para fazer a leitura dos tempos de chegada e as polaridades das ondas $\mathrm{P}$ e $\mathrm{S}$.

Os hipocentros foram determinados com o programa HYPO71 (Lee \& Lahr, 1975) que tem sido utilizado com sucesso no estudo da sismicidade no Nordeste do Brasil (Ferreira et al., 1987, 1995, 1998 e 2008; do Nascimento, 1997; Lima Neto et al. 2009).

A relocação dos hipocentros foi realizada com o algoritmo da dupla diferença HypoDD (Waldhauser, 2001).

\section{Resultados}

\section{Hipocentros}

Antes de usar o HYPO71(Lee \& Lahr, 1975) num modelo de semi-espaço, necessita-se determinar, inicialmente, os valores da velocidade das ondas $P\left(V_{P}\right)$ e a razão entre as velocidades das ondas $\mathrm{P}$ e $\mathrm{S}\left(\mathrm{k}=\mathrm{V}_{\mathrm{P}} / \mathrm{V}_{\mathrm{S}}\right)$. $\mathrm{O}$ modelo do semi-espaço foi adotado para este estudo, pois o mesmo tem sido usado com êxito no estudo da atividade sísmica do Nordeste do Brasil (Ferreira et al.,
1987, 1995, 1998 e 2008; Lima Neto et al 2009, 2010, 2011).

O modelo de velocidades foi obtido de forma conjunta, considerando o Diagrama Wadati (figura 3) como critério de consistência dos dados e escolhendo o conjunto de parâmetros com maior quantidade de sismos que tivessem as menores médias de erros. Isto foi feito variando a velocidade da onda $P\left(V_{P}\right)$ de $5,0 \mathrm{~km} / \mathrm{s}$ a 6,4 $\mathrm{km} / \mathrm{s}$ e a razão $V_{\mathrm{P}} / \mathrm{V}_{\mathrm{S}}$ de 1,60 até 1,74 . Os valores médios do erro quadrático médio, a média do erro horizontal e a média do erro vertical foram calculadas com auxilio do programa HYPO71. O melhor modelo encontrado de forma conjunta foi: $V_{P}=6,00 \mathrm{~km} / \mathrm{s}$ e $V_{P} /$ $\mathrm{V}_{\mathrm{S}}=1,70$.

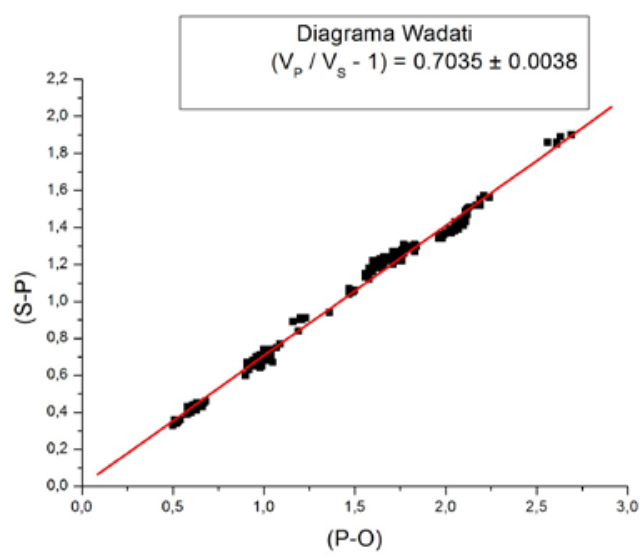

Figura 3- Diagrama Wadati dos sismos selecionados para a obtenção dos parâmetros do modelo de velocidades. $(P-O)$ é a diferença entre o tempo de chegada da onda $P$ e a hora de origem. (S-P) é a diferença entre o tempo de chegada da onda $S$ e da onda $P$.

Neste estudo o programa HYPO71 (Lee \& Lahr, 1975) foi utilizado para calcular os hipocentros, com os parâmetros que foram encontrados na escolha do modelo de velocidades (modelo do semi-espaço, $\mathrm{k}=\mathrm{V}_{\mathrm{p}} / \mathrm{V}_{\mathrm{s}}=1,70 \mathrm{e}$ $V_{P}=5,90 \mathrm{~km} / \mathrm{s}$ ) e o programa HypoDD foi utilizado para relocar os eventos.

$\mathrm{Na}$ figura 3 os eventos (erro máximo horizontal=0,3 km, erro máximo vertical $=0,3 \mathrm{~km}$, e $\mathrm{rms}$ máximo $=0.03 \mathrm{~s}$ ) registrados em pelo menos três estações são denotados por círculos amarelos, os triângulos verdes representam as estações digitais utilizadas, as linhas pretas indicam as zonas de cisalhamento mapeadas na região e as unidades geológicas são diferenciadas por cores conforme a legenda ao lado da figura 3.

Durante o período em que a rede operou foram registrados por pelo menos três estações da rede um total 64 sismos da região de Belém de Maria (eventos nos grupos 1, 2 e 3). Os resultados das análises dos dados registrados pela rede revelam que os epicentros do grupo 1, localizado próximo a estação BMLJ, não 
estão espacialmente correlacionados com a zona de cisalhamento Gravatá-Açú, assim como os eventos do grupo 2 em Agrestina (entre as estações BMUB e BMCR) não estão espacialmente correlacionados com a Zona de Cisalhamento Jurema e no grupo 3 os epicentros estão localizados na suíte intrusiva Itaporanga, onde, até o momento, não existem falhas mapeadas como mostra a figura 4

Baseado na distribuição dos epicentros e arranjo de estações não será possível obter o mecanismo focal do grupo 3, pois o mesmo se encontra fora da rede. Para os grupos 1 (cupira-PE) e 2 (Agrestina-PE) foram selecionados 15 e 8 eventos, respectivamente, que foram bem registrados em pelo menos 5 estações sismográfica.

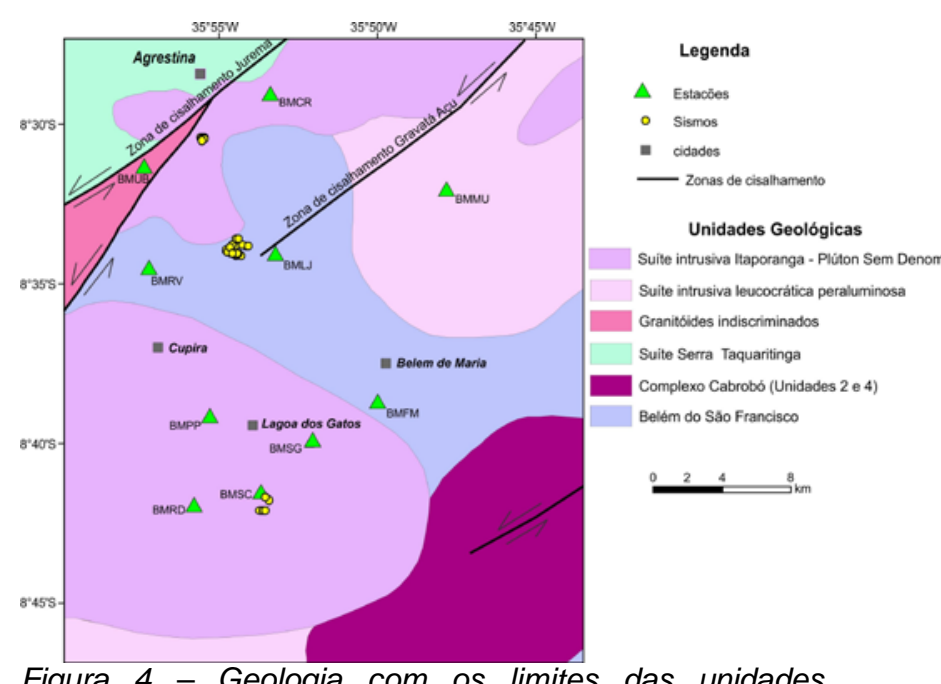

Figura 4 - Geologia com os limites das unidades litológicas que são diferenciadas pelas cores conforme a legenda ao lado da figura; as zonas de cisalhamento mapeadas estão denotadas por linhas escuras; as estações sismográficas (triângulos verdes) e epicentros (círculos amarelos).

A figura 5 mostra a correlação da forma de onda (onda $P$ ) dos 15 eventos do grupo 1 registrados pela estação BMFM que estão selecionados para serem usados no mecanismo focal dos eventos de Cupira-PE.

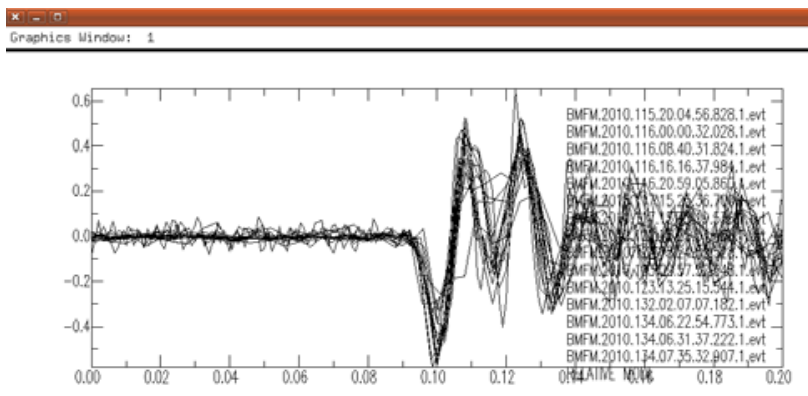

Figura 5 - A figura mostra a correlação da forma da onda $P$ de 15 eventos do grupo 1 registrados pela estação $B M F M$.

\section{Discussão e Conclusões}

Neste estudo foi mostrado que os epicentros da sismicidade registrada em São Caetano - PE no ano de 2010 não está concentrada nas áreas de Cupira-PE, Agrestina-PE e Lagoa dos Gatos não estão espacialmente correlacionados com estruturas geológicas mapeadas. A distribuição dos eventos do grupo 1 mostrados na figura $6 \mathrm{com}$ SRTM aparentam estar alinhados com a topografia da Serra Verde (Cupira$\mathrm{PE})$ numa direção diferente da observada comparada com a Zona de Cisalhamento Gravatá-Açú.

A profundidade dos sismos estudados no Distrito de Serra Verde, Cupira-PE, estudados variou entre $0,9 \mathrm{~km}$ até no máximo $3,0 \mathrm{~km}$. Enquanto os eventos de Agrestina a profundidade variou entre $1,2 \mathrm{~km}$ até $3,5 \mathrm{~km}$.

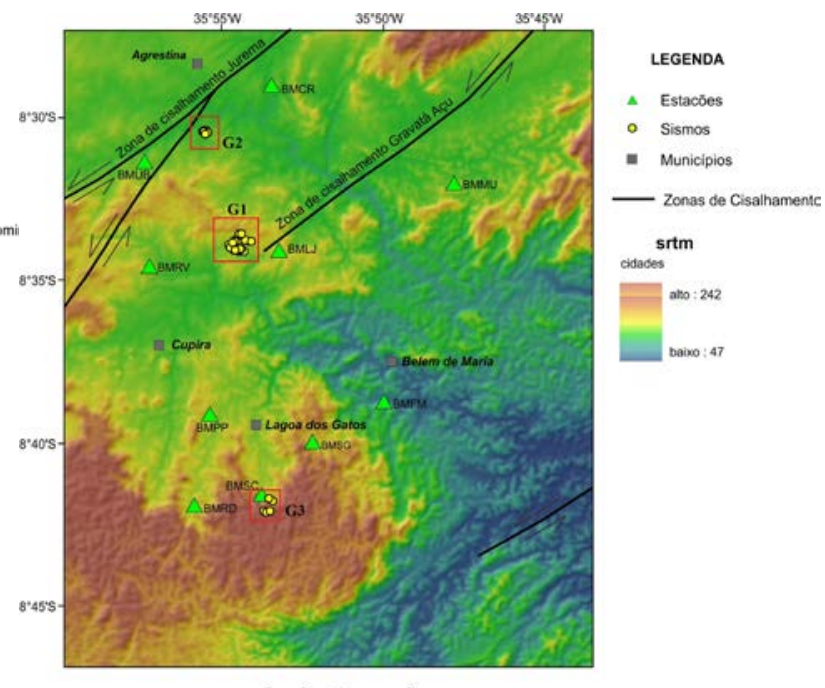

Figura 6- SRTM da região. Rede de estações BM (Belém de Maria 2010). As estações utilizadas estão representadas por triângulos verdes, as zonas de cisalhamento da região que estão mapeadas são denotadas por linhas escuras, as cidades são apontadas pelos quadrados cinzas e os retângulos vermelhos mostram as áreas onde foram registrados tremores durante a operação da rede.

A etapa seguinte deste estudo, já está em fase de execução, é a determinação dos mecanismos focal para os eventos dos grupos 1 (Cupira-PE) e 2 (Agrestina-PE). Para isso a solução proposta neste estudo levará em consideração a distribuição dos hipocentros (o mergulho e o azimute) e só foram selecionados os eventos com as polaridades de excelente qualidade das ondas $\mathrm{P}, \mathrm{a}$ amplitude da onda $\mathrm{P}$, a polaridade da onda $\mathrm{SH}$ e sua amplitudes

A área epicentral de Cupira está próxima do extremo da zona de cisalhamento Gravatá-Açú. Conforme a carta geológica do estado de Pernambuco, essa zona de cisalhamento teve, durante o ciclo brasiliano, um movimento transcorrente sinistral. Os resultados do mecanismo focal mostrarão qual o movimento atual existente e se difere em relação a zona de cisalhamento Gravatá-Açú, 
Em outras áreas que já foram estudadas no Lineamento Pernambuco, por exemplo, Caruaru em 1991 (Ferreira et al. 1998), Belo Jardim 2004 (Lopes et al 2010) e São Caetano 2010 (Lima Neto et al 2011) a atividade sísmica mostrou ter uma clara correlação geométrica com o Lineamento Pernambuco; assim como as atividades sísmicas estudas a norte do Lineamento Pernambuco, como em Caruaru 2002 (Ferreira et al 2008) e São Caetano 2007 (Lima Neto et al 2009) estavam correlacionadas em cada caso com ramificações do Lineamento Pernambuco. Esse tipo de correlação entre feições geológicas mapeadas e sismicidade no nordeste brasileiro é rara, de um modo geral, mas bastante freqüente na atividade sísmica relacionada com 0 Lineamento Pernambuco e suas ramificações.

O mecanismo focal das áreas apresentadas no atual estudo, o primeiro a ser realizado numa área ao sul do Lineamento Pernambuco, poderá confirmar ou não se esse tipo de correlação entre sismicidade e feições geológicas mapeadas é tão frequente como mostrado em estudos anteriores.

\section{Agradecimentos}

Ao Dr. Reinhardt Fuck, coordenador do Projeto INCT de Estudos Tectônicos, pelo apoio contínuo.

Ao Dr. Darcy Nascimento, coordenador do PEGBR (Pool de Equipamentos Geofísicos do Brasil).

Ao Programa de Pós-Graduação em Geodinâmica e Geofísica (PPGG) da UFRN.

A CAPES pela Bolsa de Doutorado.

\section{Referências}

Assumpção, M. 1992. The Regional intraplate stress field in South América. J. Geophys.Res.,97(B8): 11889-11903.

Dayvison,I., McCarthy,M., Powell,D., Torres,H.H., F.,Santos,C.A. 1995. Laminar flow in shear zones: the Pernambuco Shear Zone,NE Brazil.Journal of Structural Geology 17: 149-161.

do Nascimento, A .F. 1997. Estudo da sismicidade induzida pelo reservatório da Barragem do Assu (RN)Tese de mestrado, 001-PPGG/UFRN ,68 pp.

Ferreira, J.M. \& Assumpção, M. 1983. Sismicidade do Nordeste do Brasil. Rev Bras. Geofís, 1: 67-88.

Ferreira, J.M., Takeia, M., Costa, J.M., Moreira, J.A., Assumpção, M., Veloso, J.A.V. \& Pearce, R.G. 1987. A continuing intraplate earthquake sequence near João Câmara, Northeastern Brazil - Preliminary results. Gephys.Res.Lett., 14: 1402-1405.

Ferreira, J.M., Assumpção, M., Costa, J.M., Moreira, J.A.M., Pearce, R.G. \& Takeya, M.K.1995. Correlation of seimicity and water level in the Açu reseivor- na example from Northeast Brazil. Bull. Seism.Soc, Am, 85: 14831489.
Ferreira, J.M., Oliveira, R.T., Takeya, M.K. \& Assumpção, M. 1998. Superposition of local and regional stress in northeast Brazil: evidence from focal mechanism around the Potiguar marginal basin. Geophys. J. Int., 134: 341355.

Ferreira, J.M., Bezerra, F.H.R., Souza, M.O.L., do Nascimento, A.F., Martins Sá, J., França, G.S. 2008. The role of precambrian mylonitic na present day stress field in the coseismic reactivation of the Pernambuco Lineament, Brazil. Tectonophysics. 456:11-126.

Lee, W.H.K. \& Lahr, J.C. 1975. HYPO71 (revised): a computer program for determining hypocenter, magnitude and first motion pattern of local earthquakes. U.S. Geol. Surv. Open File Rep. 75-311, 114 pp.

Lima Neto, H.C., Ferreira, J.M., Nascimento, A.F. \& Bezerra, H.R., 2009.Estudo da atividade sísmica em São Caetano-PE em 2007, 11th International Congress of the Brazilian Geophysical Society, Salvador, Extended Abstract, $6 \mathrm{pp}$.

Lima Neto, H.C., Ferreira, J.M., Nascimento, A.F. Assumpção, M, Bezerra, H.R. \& Menezes, E.A.S. 2011. Uma nova área sísmica em São Caetano-PE e sua correlação com o Lineamento pernambuco, 12th International Congress of the Brazilian Geophysical Society, Rio de Janeiro, Extended Abstract, 5 pp.

Lopes, A.E.V.; Assumpção, M.; do Nascimento, A.F.; Ferreira, J.M.; Menezes, E.A.S. \& Barbosa, J.R. (2010), Intraplate Earthquake Swarm in Belo Jardim, NE Brazil: Reactivation of a Major NeoProterozoic Shear Zone (Pernambuco Lineament), Geophys. J. Int., 180(3), 13031312

Tapley W.C. \& Tull, J.E. 1991. SAC - Seismic Analysis Code. USER MANUAL, Livermore National Laboratory, $413 \mathrm{pp}$.

Waldhauser, F., 2001. HypoDD - A Program to Compute Double-Difference Hypocenter Locations, U.S. Geol. Survey, Open File Report 01-113. 